\title{
On the characterization of $D$-preinvex functions
}

Taiyong $\mathrm{Li}^{1 *}$ and Min Huang ${ }^{2}$

"Correspondence:

litaiyong781130@163.com

1 Tianmu College, Zhejiang A \& F

University, Hangzhou, 311300,

China

Full list of author information is

available at the end of the article

\begin{abstract}
In (J. Inequal. Appl. 2006:9532, 2006), Peng and Zhu discussed interrelations among $D$-preinvexity, $D$-semistrict preinvexity, and D-strict preinvexity for vector-valued functions. In this note, we show that the same results or even more general ones can be obtained under weaker assumptions. We also give a new characterization of $D$-preinvexity and $D$-semistrict preinvexity under mild conditions.
\end{abstract}

MSC: $90 C 26$

Keywords: D-preinvex functions; D-semistrictly preinvex functions; $D$-strictly preinvex functions; semicontinuity

\section{Introduction}

Convexity and some generalizations of convexity play a crucial role in mathematical economics, engineering, management science, and optimization theory. Therefore, it is important to consider wider classes of generalized convex functions and also to seek practical criteria for convexity or generalized convexity (see Refs. [1-9] and the references therein). A significant generalization of convex functions is the introduction of preinvex functions, which is due to Ben and Mond [4]. Yang and Li [1] presented some properties of preinvex functions; in [2] they introduced two new classes of generalized convex functions called semistrictly preinvex functions and strictly preinvex functions. They established relationships between preinvex functions and semistrictly preinvex functions under a certain set of conditions. Very recently, Peng and Zhu [7] introduced the vector cases of strict preinvexity and semistrict preinvexity and established some relations between them. In this paper, we show that the same results or even a generalized version of their results can be obtained under weaker assumptions. Moreover, we give a new characterization of $D$-preinvexity and $D$-semistrict preinvexity under mild conditions. The outline of the paper is as follows. In Section 2, we give some preliminaries. The main results of the paper are presented in Section 3.

\section{Preliminaries}

Throughout this paper, we will use the following assumptions. Let $X$ be a real topological vector space and $Y$ be a real locally convex vector space, let $K \subset X$ be a nonempty subset. Let $D \subset Y$ be a nonempty pointed closed convex cone and $Y^{*}$ be the dual space of $Y$. The dual cone $D^{*}$ of cone $D$ is defined by

$$
D^{*}=\left\{k^{*} \in Y^{*}:\left\langle k^{*}, x\right\rangle \geq 0, \forall x \in D\right\} .
$$

C 2012 Li and Huang; licensee Springer. This is an Open Access article distributed under the terms of the Creative Commons Attribution License (http://creativecommons.org/licenses/by/2.0), which permits unrestricted use, distribution, and reproduction in any medium, provided the original work is properly cited. 
From the bipolar theorem, we have the following

Lemma 2.1 For all $q \in D^{*},\langle q, d\rangle \geq 0$ if and only if $d \in D$.

Now we will describe some definitions of generalized convexity.

Definition 2.1 ([3]) Let the set $K \subseteq X$ and the vector function $\eta: X \times X \rightarrow X$ be given. $K$ is said to be an invex set with respect to the function $\eta$ iff

$$
\forall x, y \in K, \forall \lambda \in[0,1] \Rightarrow y+\lambda \eta(x, y) \in K
$$

Definition 2.2 ([7]) Let $K \subseteq X$ be an invex set with respect to $\eta: X \times X \rightarrow X$. The vectorvalued function $f: K \rightarrow Y$ is said to be $D$-preinvex on $K$ iff, $\forall x, y \in K, \forall \lambda \in(0,1)$,

$$
f(y+\lambda \eta(x, y)) \in \lambda f(x)+(1-\lambda) f(y)-D
$$

Definition 2.3 ([7]) Let $K \subseteq X$ be an invex set with respect to $\eta: X \times X \rightarrow X$. The vectorvalued function $f: K \rightarrow Y$ is said to be $D$-semistrictly preinvex on $K$ iff, $\forall x, y \in K$ such that $f(x) \neq f(y)$ and $\forall \lambda \in(0,1)$,

$$
f(y+\lambda \eta(x, y)) \in \lambda f(x)+(1-\lambda) f(y)-\operatorname{int} D
$$

Definition 2.4 ([7]) Let $K \subseteq X$ be an invex set with respect to $\eta: X \times X \rightarrow X$. The function $f: K \rightarrow Y$ is said to be $D$-strictly preinvex on $K$ iff, $\forall x, y \in K, x \neq y, \forall \lambda \in(0,1)$,

$$
f(y+\lambda \eta(x, y)) \in \lambda f(x)+(1-\lambda) f(y)-\operatorname{int} D .
$$

In [10], Jeyakumar et al. introduced the $*$-lower semicontinuity for a vector-valued function as follows.

Definition 2.5 ([10]) The vector-valued function $f: K \rightarrow Y$ is $*$-lower semicontinuous if for every $q \in D^{*}, q(f)(\cdot)=\langle q, f(\cdot)\rangle$ is lower semicontinuous on $K$.

In [7], Peng et al. introduced the $*$-upper semicontinuity for a vector-valued function as follows.

Definition 2.6 ([7]) The vector-valued function $f: K \rightarrow Y$ is $*$-upper semicontinuous if for every $q \in D^{*}, q(f)(\cdot)=\langle q, f(\cdot)\rangle$ is upper semicontinuous on $K$.

In order to prove our main result, we need Condition C introduced by Mohan and Neogy [11] as follows.

Condition C Let $\eta: X \times X \rightarrow X$. We say that the function $\eta$ satisfies Condition $\mathrm{C}$ iff, $\forall x, y \in K, \forall \lambda \in[0,1]$

$$
\begin{aligned}
& \eta(y, y+\lambda \eta(x, y))=-\lambda \eta(x, y), \\
& \eta(x, y+\lambda \eta(x, y))=(1-\lambda) \eta(x, y) .
\end{aligned}
$$




\section{Properties of $D$-preinvex functions}

In this section, we assume always that:

(i) $K \subseteq X$ is a nonempty invex set with respect to $\eta: X \times X \rightarrow X$;

(ii) $\eta$ satisfies Condition $C$; $f$ is a vector-valued function on $K$.

The following result was proved in Ref. [7]; see Theorem 2.2 in Ref. [7].

Theorem 3.1 Let $K$ be a nonempty open invex set in $X$ with respect to $\eta: X \times X \rightarrow X$. If $f: K \rightarrow Y$ is $*$-upper semicontinuous and satisfies $f(y+\eta(x, y)) \in f(x)-D, \forall x, y \in K$, then $f$ is a D-preinvex function for the same $\eta$ on $K$ if and only if there exists an $\alpha \in(0,1)$ such that

$$
f(y+\alpha \eta(x, y)) \in \alpha f(x)+(1-\alpha) f(y)-D, \quad \forall x, y \in K
$$

Now we improve the above theorem as follows.

Theorem 3.2 Let $f: K \rightarrow Y$ be $*$-upper semicontinuous and satisfy $f(y+\eta(x, y)) \in f(x)-D$, $\forall x, y \in K$, then $f$ is a D-preinvex function for the same $\eta$ on $K$ if and only if there exists an $\alpha \in(0,1)$ such that

$$
f(y+\alpha \eta(x, y)) \in \alpha f(x)+(1-\alpha) f(y)-D, \quad \forall x, y \in K
$$

Proof The necessity follows directly from the definition of $D$-preinvexity for the vectorvalued function $f$. We only need to prove the sufficiency. By Lemma 2.1 in Ref. [7], the set $A=\{\alpha \in[0,1] \mid f(y+\alpha \eta(x, y)) \in \alpha f(x)+(1-\alpha) f(y)-D, \forall x, y \in K\}$ is dense in the interval $[0,1]$. Then $\forall \bar{\alpha} \in(0,1), \exists\left\{\alpha_{n}\right\} \subseteq(0,1) \cap A$ such that $\alpha_{n}<\bar{\alpha}$ for each $n$ and $\alpha_{n} \rightarrow \bar{\alpha}$, as $n \rightarrow \infty$. Give $x, y \in K$, denote

$$
z=y+\bar{\alpha} \eta(x, y)
$$

Define, for each $n$,

$$
y_{n}=y+\frac{\bar{\alpha}-\alpha_{n}}{1-\alpha_{n}} \eta(x, y) .
$$

Thus,

$$
y_{n} \rightarrow y, \quad n \rightarrow \infty
$$

Since $0<\alpha_{n}<\bar{\alpha}<1$, we have

$$
0<\frac{\bar{\alpha}-\alpha_{n}}{1-\alpha_{n}}<1
$$

which in turn implies that $y_{n} \in K$, by (3.2) and $K$ is invex with respect to $\eta$. From Condition $\mathrm{C}$, we have

$$
\begin{aligned}
y_{n}+\alpha_{n} \eta\left(x, y_{n}\right) & =y+\frac{\bar{\alpha}-\alpha_{n}}{1-\alpha_{n}} \eta(x, y)+\alpha_{n} \eta\left(x, y+\frac{\bar{\alpha}-\alpha_{n}}{1-\alpha_{n}} \eta(x, y)\right) \\
& =y+\frac{\bar{\alpha}-\alpha_{n}}{1-\alpha_{n}} \eta(x, y)+\alpha_{n}\left(1-\frac{\bar{\alpha}-\alpha_{n}}{1-\alpha_{n}}\right) \eta(x, y)=y+\bar{\alpha} \eta(x, y)=z .
\end{aligned}
$$


As $\alpha_{n} \in A$, we have

$$
f(z)=f(y+\bar{\alpha} \eta(x, y))=f\left(y_{n}+\alpha_{n} \eta\left(x, y_{n}\right)\right) \in \alpha_{n} f(x)+\left(1-\alpha_{n}\right) f\left(y_{n}\right)-D
$$

By the $*$-upper semicontinuity of $f$ on $K$, for every $q \in D^{*}, q(f)(\cdot)$ is upper semicontinuous, it follows that for any $\epsilon>0$, there exists an $N>0$ such that the following holds:

$$
q(f)\left(y_{n}\right) \leq q(f)(y)+\epsilon, \quad \forall n>N
$$

Hence,

$$
\begin{aligned}
q(f)(z) & \leq \alpha_{n} q(f)(x)+\left(1-\alpha_{n}\right) q(f)\left(y_{n}\right) \\
& \leq \alpha_{n} q(f)(x)+\left(1-\alpha_{n}\right)(q(f)(y)+\epsilon) \\
& \rightarrow \bar{\alpha} q(f)(x)+(1-\bar{\alpha})(q(f)(y)+\epsilon) \quad(n \rightarrow \infty) .
\end{aligned}
$$

Since $\epsilon>0$ may be arbitrarily small, then for all $q \in D^{*}$, we have

$$
q(f)(z) \leq \bar{\alpha} q(f)(x)+(1-\bar{\alpha}) q(f)(y)
$$

Since $q$ is linear, by Lemma 2.1, we have

$$
f(z) \in \bar{\alpha} f(x)+(1-\bar{\alpha}) f(y)-D .
$$

Hence, $f$ is a $D$-preinvex function for the same $\eta$ on $K$, this completes the proof.

Remark 3.1 We see from Theorem 3.2 that the condition of openness in Theorem 3.1 can be deleted in order to obtain the same results.

Now, we state another result in Ref. [7]; see Theorem 3.3 in Ref. [7].

Theorem 3.3 Let $f$ be a D-preinvex function on $K$. If there exists an $\alpha \in(0,1)$ such that, for each pair $x, y \in K, x \neq y$,

$$
f(y+\alpha \eta(x, y)) \in \alpha f(x)+(1-\alpha) f(y)-\operatorname{int} D, \quad \forall x, y \in K
$$

then $f$ is a D-strictly preinvex function on $K$.

The above theorem can be improved as follows.

Theorem 3.4 Letf be a D-preinvex function with respect to $\eta: X \times X \rightarrow X$ on $K$. For each pair $x, y \in K, x \neq y$, if there exists an $\alpha \in(0,1)$ such that

$$
f(y+\alpha \eta(x, y)) \in \alpha f(x)+(1-\alpha) f(y)-\operatorname{int} D, \quad \forall x, y \in K
$$

then $f$ is a strictly D-preinvex function on $K$. 
Proof By contradiction, suppose that there exist $x, y \in K, x \neq y, \lambda \in(0,1)$ such that

$$
\lambda f(x)+(1-\lambda) f(y)-f(y+\lambda \eta(x, y)) \notin \operatorname{int} D .
$$

Denote

$$
z=y+\lambda \eta(x, y)
$$

Since $f$ is $D$-preinvex, we have

$$
\lambda f(x)+(1-\lambda) f(y)-f(y+\lambda \eta(x, y)) \in D .
$$

We note that the pair $x, z$ and the pair $z, y$ are both distinct under condition (3.5). There exist $\beta_{1}, \beta_{2} \in(0,1)$ such that

$$
\begin{aligned}
& \beta_{1} f(x)+\left(1-\beta_{1}\right) f(z)-f\left(z+\beta_{1} \eta(x, z)\right) \in \operatorname{int} D, \\
& \beta_{2} f(z)+\left(1-\beta_{2}\right) f(y)-f\left(y+\beta_{2} \eta(z, y)\right) \in \operatorname{int} D .
\end{aligned}
$$

Denote

$$
\bar{x}=z+\beta_{1} \eta(x, z), \quad \bar{y}=y+\beta_{2} \eta(z, y) .
$$

From Condition C,

$$
\begin{aligned}
\bar{x} & =z+\beta_{1} \eta(x, z)=y+\lambda \eta(x, y)+\beta_{1} \eta(x, y+\lambda \eta(x, y)) \\
& =y+\lambda \eta(x, y)+(1-\lambda) \beta_{1} \eta(x, y) \\
& =y+\left(\lambda+(1-\lambda) \beta_{1}\right) \eta(x, y), \\
\bar{y} & =y+\beta_{2} \eta(z, y)=y+\beta_{2} \eta(y+\lambda \eta(x, y), y) \\
& =y+\beta_{2} \eta(y+\lambda \eta(x, y), y+\lambda \eta(x, y)-\lambda \eta(x, y)) \\
& =y+\beta_{2} \eta(y+\lambda \eta(x, y), y+\lambda \eta(x, y)+\eta(y, y+\lambda \eta(x, y))) \\
& =y-\beta_{2} \eta(y, y+\lambda \eta(x, y)) \\
& =y+\lambda \beta_{2} \eta(x, y) .
\end{aligned}
$$

Let $\mu_{1}=\lambda+(1-\lambda) \beta_{1}, \mu_{2}=\lambda \beta_{2}, \mu=\frac{\lambda-\mu_{2}}{\mu_{1}-\mu_{2}}$. It is easy to verify that $\mu_{1}, \mu_{2}, \mu \in(0,1)$. Again from Condition $\mathrm{C}$,

$$
\begin{aligned}
\bar{y}+\mu \eta(\bar{x}, \bar{y})= & y+\mu_{2} \eta(x, y)+\mu \eta\left(y+\mu_{1} \eta(x, y), y+\mu_{2} \eta(x, y)\right) \\
= & y+\mu_{2} \eta(x, y)+\mu \eta\left(y+\mu_{1} \eta(x, y), y+\mu_{1} \eta(x, y)+\left(\mu_{2}-\mu_{1}\right) \eta(x, y)\right) \\
= & y+\mu_{2} \eta(x, y)+\mu \eta\left(y+\mu_{1} \eta(x, y), y+\mu_{1} \eta(x, y)\right. \\
& \left.+\left(\frac{\mu_{2}-\mu_{1}}{1-\mu_{1}}\right) \eta\left(x, y+\mu_{1} \eta(x, y)\right)\right)
\end{aligned}
$$




$$
\begin{aligned}
& =y+\mu_{2} \eta(x, y)-\left(\frac{\mu\left(\mu_{2}-\mu_{1}\right)}{1-\mu_{1}}\right) \eta\left(x, y+\mu_{1} \eta(x, y)\right) \\
& =y+\mu_{2} \eta(x, y)-\mu\left(\mu_{2}-\mu_{1}\right) \eta(x, y) \\
& =y+\left(\mu_{2}-\mu\left(\mu_{2}-\mu_{1}\right)\right) \eta(x, y) \\
& =y+\lambda \eta(x, y) \\
& =z .
\end{aligned}
$$

Since $f$ is $D$-preinvex, we have

$$
\mu f(\bar{x})+(1-\mu) f(\bar{y})-f(z) \in D .
$$

Thus, from (3.7)-(3.10), we have

$$
\begin{aligned}
\mu f(\bar{x})+ & (1-\mu) f(\bar{y})-f(z)+\mu\left(\beta_{1} f(x)+\left(1-\beta_{1}\right) f(z)-f(\bar{x})\right) \\
& +(1-\mu)\left(\beta_{2} f(z)+\left(1-\beta_{2}\right) f(y)-f(\bar{y})\right) \in \operatorname{int} D \\
\Rightarrow \quad & \mu \beta_{1} f(x)+\left(\mu\left(1-\beta_{1}\right)+(1-\mu) \beta_{2}\right) f(z)+(1-\mu)\left(1-\beta_{2}\right) f(y)-f(z) \in \operatorname{int} D \\
\Rightarrow \quad & \mu \beta_{1} f(x)+\left(\mu\left(1-\beta_{1}\right)+(1-\mu) \beta_{2}\right)(\lambda f(x)+(1-\lambda) f(y)) \\
& +(1-\mu)\left(1-\beta_{2}\right) f(y)-f(z) \in \operatorname{int} D \\
\Rightarrow \quad & \left(\mu \beta_{1}+\lambda \mu\left(1-\beta_{1}\right)+\lambda \beta_{2}(1-\mu)\right) f(x)+\left((1-\mu)\left(1-\beta_{2}\right)\right. \\
& \left.+(1-\lambda) \mu\left(1-\beta_{1}\right)+(1-\lambda) \beta_{2}(1-\mu)\right) f(y)-f(z) \in \operatorname{int} D \\
\Rightarrow \quad & \lambda f(x)+(1-\lambda) f(y)-f(z) \in \operatorname{int} D .
\end{aligned}
$$

Where

$$
\begin{aligned}
& \mu \beta_{1}+\lambda \mu\left(1-\beta_{1}\right)+\lambda \beta_{2}(1-\mu) \\
& \quad=\mu\left(\lambda+\beta_{1}(1-\lambda)-\lambda \beta_{2}\right)+\lambda \beta_{2} \\
& \quad=\mu\left(\lambda+\beta_{1}(1-\lambda)-\mu_{2}\right)+\mu_{2} \\
& \quad=\mu\left(\mu_{1}-\mu_{2}\right)+\mu_{2} \\
& \quad=\lambda, \\
& (1-\mu)\left(1-\beta_{2}\right)+(1-\lambda) \mu\left(1-\beta_{1}\right)+(1-\lambda) \beta_{2}(1-\mu) \\
& \quad=(1-\mu)\left(1-\mu_{2}\right)+\mu\left(1-\mu_{1}\right) \\
& \quad=1-\mu_{2}+\mu\left(\mu_{2}-\mu_{1}\right) \\
& \quad=1-\lambda,
\end{aligned}
$$

which contradicts (3.6). This completes the proof.

Remark 3.2 In Theorem 3.3, a uniform $\alpha \in(0,1)$ is needed, while in Theorem 3.4 this condition has been weakened to great extent.

By Theorem 2.3 in Ref. [7] and Theorem 3.4 above, we have the following corollary. 
Corollary 3.1 Let $f$ be $a *$-lower semicontinuous function on $K$ and satisfy $f(y+\eta(x, y)) \in$ $f(x)-D, \forall x, y \in K$. For each pair $x, y \in K, x \neq y$, if there exists an $\alpha \in(0,1)$ such that

$$
f(y+\alpha \eta(x, y)) \in \alpha f(x)+(1-\alpha) f(y)-\operatorname{int} D, \quad \forall x, y \in K
$$

then $f$ is a strictly D-preinvex function on $K$.

Corollary 3.2 Let $f$ be a $*$-upper semicontinuous function on $K$ and satisfy $f(y+\eta(x, y)) \in$ $f(x)-D, \forall x, y \in K$. If there exists an $\alpha \in(0,1)$ such that, for each pair $x, y \in K, x \neq y$,

$$
f(y+\alpha \eta(x, y)) \in \alpha f(x)+(1-\alpha) f(y)-\operatorname{int} D, \quad \forall x, y \in K
$$

then $f$ is a strictly D-preinvex function on $K$.

Proof This result is obtained by Theorems 3.2 and 3.4 above.

Theorem 3.5 Let $f$ be $a *$-lower semicontinuous function on $K$ and satisfy $f(y+\eta(x, y)) \in$ $f(x)-D, \forall x, y \in K$. If there exists an $\alpha \in(0,1)$ such that, for each pair $x, y \in K, f(x) \neq f(y)$ implies

$$
f(y+\alpha \eta(x, y)) \in \alpha f(x)+(1-\alpha) f(y)-\operatorname{int} D, \quad \forall x, y \in K,
$$

then $f$ is both a D-preinvex function on $K$ and a D-semistrictly preinvex function on $K$.

Proof First, we prove that $f$ is a $D$-preinvex function on $K$. By Theorem 2.3 in Ref. [7], we need to show that for each $x, y \in K$, there exists $\lambda \in(0,1)$ such that

$$
f(y+\lambda \eta(x, y)) \in \lambda f(x)+(1-\lambda) f(y)-D
$$

Assume, by contradiction, that there exists $x, y \in K$ such that

$$
f(y+\lambda \eta(x, y)) \notin \lambda f(x)+(1-\lambda) f(y)-D, \quad \forall \lambda \in(0,1) .
$$

If $f(x) \neq f(y)$, condition (3.11) implies

$$
f(y+\alpha \eta(x, y)) \in \alpha f(x)+(1-\alpha) f(y)-\operatorname{int} D \subset \alpha f(x)+(1-\alpha) f(y)-D,
$$

which contradicts (3.12). Thus, we have $f(x)=f(y)$, and then (3.12) implies

$$
f(y+\lambda \eta(x, y)) \notin f(x)-D=f(y)-D, \quad \forall \lambda \in(0,1) .
$$

Since $D$ is a closed convex pointed cone, by the strong separation theorem for a convex set, it follows that there exists $k^{*} \in D^{*} \backslash\{0\}$ such that

$$
\begin{aligned}
& \left\langle k^{*}, \lambda f(x)+(1-\lambda) f(y)-f(y+\lambda \eta(x, y))\right\rangle \\
& =\left\langle k^{*}, f(y)-f(y+\lambda \eta(x, y))\right\rangle<0, \quad \forall \lambda \in(0,1) .
\end{aligned}
$$


Let $\lambda=\frac{\alpha-1}{\alpha-2} \in(0,1)$, and let $z=y+\lambda \eta(x, y)$, then the above inequality reduces to

$$
\left\langle k^{*}, f(x)-f(z)\right\rangle<0,
$$

and

$$
\left\langle k^{*}, f(y)-f(z)\right\rangle<0 \text {. }
$$

Define

$$
z_{1}=z+\alpha \eta(x, z), \quad z_{2}=z_{1}+\alpha \eta\left(y, z_{1}\right) .
$$

From Condition C, we obtain

$$
\begin{aligned}
z_{1}= & y+\lambda \eta(x, y)+\alpha \eta(x, y+\lambda \eta(x, y)) \\
& =y+\lambda \eta(x, y)+\alpha(1-\lambda) \eta(x, y) \\
& =y+(\alpha+\lambda(1-\alpha)) \eta(x, y) \\
& =y+\frac{1}{2-\alpha} \eta(x, y), \\
z_{2} & =y+\frac{1}{2-\alpha} \eta(x, y)+\alpha \eta\left(y, y+\frac{1}{2-\alpha} \eta(x, y)\right) \\
& =y+\left(\frac{1}{2-\alpha}+\frac{-\alpha}{2-\alpha}\right) \eta(x, y) \\
& =y+\frac{1-\alpha}{2-\alpha} \eta(x, y) \\
& =y+\lambda \eta(x, y) \\
& =z .
\end{aligned}
$$

Since $\frac{1}{2-\alpha} \in(0,1)$, it follows from (3.14) that

$$
\left\langle k^{*}, f(y)-f\left(z_{1}\right)\right\rangle<0 \text {. }
$$

Conditions (3.11) and (3.13) give

$$
f\left(z_{1}\right)=f(z+\alpha \eta(x, z)) \in \alpha f(x)+(1-\alpha) f(y)-\operatorname{int} D .
$$

It follows that

$$
\left\langle k^{*}, \alpha f(x)+(1-\alpha) f(z)-f\left(z_{1}\right)\right|>0,
$$

which together with (3.15) yields

$$
\left\langle k^{*}, f(z)-f\left(z_{1}\right)\right\rangle>0 .
$$


From (3.17) and condition (3.11), we get

$$
f\left(z_{2}\right)=f\left(z_{1}+\alpha \eta\left(y, z_{1}\right)\right) \in \alpha f(y)+(1-\alpha) f\left(z_{1}\right)-\operatorname{int} D .
$$

It follows that

$$
\left\langle k^{*}, \alpha f(y)+(1-\alpha) f\left(z_{1}\right)-f\left(z_{2}\right)\right\rangle>0,
$$

which together with (3.17) yields

$$
\left\langle k^{*}, f\left(z_{1}\right)-f\left(z_{2}\right)\right\rangle=\left\langle k^{*}, f\left(z_{1}\right)-f(z)\right|>0,
$$

which contradicts (3.18), hence $f$ is a $D$-preinvex function on $K$. Next, the $D$-semistrict preinvexity of $f$ on $K$ follows from Theorem 3.9 of Ref. [7].

\section{Conclusions}

In this paper, we firstly obtain a property of $D$-preinvex functions. We then get a sufficient condition of the strictly $D$-preinvex functions in terms of intermediate-point $D$ preinvex functions. We finally obtain a sufficient condition of $D$-preinvex functions and $D$-semistrictly preinvex functions. Our results improve and extend the existing ones in the literature.

Competing interests

The authors declare that they have no competing interests.

\section{Authors' contributions}

The main theorems are proved by TL. Both authors drafted the manuscript, read and approved the final manuscript.

\section{Author details}

${ }^{1}$ Tianmu College, Zhejiang A \& F University, Hangzhou, 311300, China. ${ }^{2}$ School of Economy and Management, Zhejiang A \& F University, Hangzhou, 311300, China

\section{Acknowledgements}

The research of the authors is partially supported by the Foundation of Department of Education of Zhejiang Province under Grant Y201121204 and by the Natural Science Foundation of Zhejiang Province (Y7100544). The authors would like to express their thanks to the referees for helpful suggestions.

Received: 10 April 2012 Accepted: 4 October 2012 Published: 17 October 2012

\section{References}

1. Yang, XM, Li, D: On properties of preinvex functions. J. Math. Anal. Appl. 256, 229-241 (2001)

2. Yang, $X M$, Li, D: Semistrictly preinvex functions. J. Math. Anal. Appl. 258, 287-308 (2001)

3. Weir, T, Mond, B: Pre-invex functions in multiple objective optimization. J. Math. Anal. Appl. 136, $29-38$ (1988)

4. Ben-Israel, A, Mond, B: What is invexity? J. Aust. Math. Soc. Ser. B, Appl. Math 28, 1-9 (1986)

5. Antczak, T: r-pre-invexity and r-invexity in mathematical programming. Comput. Math. Appl. 50, $551-566$ (2005)

6. Antczak, T: New optimality conditions and duality results of G-type in differentiable mathematical programming. Nonlinear Anal. 66, 1617-1632 (2007)

7. Peng, JW, Zhu, DL: On D-preinvex-type functions. J. Inequal. Appl. 2006, 93532 (2006)

8. Yang, $X M$, Yang, $X Q$, Teo, KL: Characterizations and applications of prequasi-invex functions. J. Optim. Theory Appl. $110,645-668(2001)$

9. Pini, R: Invexity and generalized convexity. Optimization 22, 513-525 (1991)

10. Jeyakumar, V, Oettli, W, Natividad, M: A solvability theorem for a class of quasiconvex mappings with applications to optimization. J. Math. Anal. Appl. 179(2), 537-546 (1993)

11. Mohan, SR, Neogy, SK: On invex sets and preinvex functions. J. Math. Anal. Appl. 189, $901-908$ (1995)

doi:10.1186/1029-242X-2012-240

Cite this article as: Li and Huang: On the characterization of D-preinvex functions. Journal of Inequalities and Applications 2012 2012:240. 